\title{
Understanding Palliative Cancer Chemotherapy: About Shared Decisions and Shared Trajectories
}

\author{
Susanne J. de Kort · Jeannette Pols · Dick J. Richel • \\ Nelleke Koedoot · Dick L. Willems
}

Published online: 6 May 2009

(C) The Author(s) 2009. This article is published with open access at Springerlink.com

\begin{abstract}
Most models of patient-physician communication take decision-making as a central concept. However, we found that often the treatment course of metastatic cancer patients is not easy to describe in straightforward terms used in decisionmaking models but is instead frequently more erratic. Our aim was to analyse these processes as trajectories. We used a longitudinal case study of 13 patients with metastatic colorectal and pancreatic cancer for whom palliative chemotherapy was a treatment option, and analysed 65 semi-structured interviews. We analysed three characteristics of the treatment course that contributed to the 'erraticness' of the course: (1) The treatment (with or without chemotherapy) contained many options; (2) these options were not stable entities to be decided upon, but changed identity over the course of treatment, and (3) contrary to the closure (option X means no option $\mathrm{Y}$, $\mathrm{Z}$, etc.) a decision implies, the treatment course was a continuous process in which options instead remained open. When the treatment course is characterised by these many and changeable options that do not result in closure, the shared decision-making model should take these into account. More attention needs to be paid to the erratic character of the process in which the doctor has to provide continuous information that is related to the changing situation of the patient; also, flexibility in dealing with protocols is warranted, as is vigilance about the overall direction of the process.
\end{abstract}

S. J. de Kort $(\bowtie) \cdot$ J. Pols · D. L. Willems

Section Medical Ethics, Department of General Practise, Division of Clinical Methods \& Public

Health, Academic Medical Centre, University of Amsterdam, Meibergdreef 9, 1100 DD

Amsterdam, The Netherlands

e-mail: s.j.dekort@amc.uva.nl; susannedekort@hotmail.com

\section{J. Richel}

Department of Internal Medicine, Academic Medical Centre, University of Amsterdam, Amsterdam, The Netherlands

\section{N. Koedoot}

Department of Medical Psychology, Academic Medical Center, University of Amsterdam, Amsterdam, The Netherlands 
Keywords Palliative cancer chemotherapy · Shared decision-making ·

Qualitative research · Medical ethics

\section{Introduction}

Different models of patient-physician communication exist. Paternalistic models, on the one extreme, put the decision on the side of the physician, counselling models, on the other, imply the picture of a patient making autonomous decisions on the basis of proper information, and in-between, shared decision-making models view making health care decisions as the common task of the physician and patient $[2,7]$. However, the concept of decision-making plays a central role in these models. The dictionary meaning of decision is: 'a resolution or conclusion reached after consideration'.

We investigated the process by which oncologists and patients shape the treatment course in metastatic cancer. Decisions in the sense given above were often hard to find. Therefore, we question the idea that making a decision is the central activity for understanding the course of treatment for metastatic cancer. Experienced clinicians will not be surprised that in our study of the practise of metastatic cancer treatment we found the treatment course to be quite erratic. Everyday clinicians' experience makes clear that the treatment course is indeed continually being revised as the patient's response, experience with treatment, and their disease changes over time. However, not much is known about this process because, as far as we know, it has not been investigated and articulated by empirical research before. We will propose to call this process 'shared trajectory making'.

We formulated the following research question: what characterises the course of treatment for metastatic cancer that leads to starting, foregoing, continuing or discontinuing, modifying, or switching palliative chemotherapy? To answer this question, we observed outpatient physician-patient contacts and conducted subsequent interviews with patients with metastatic colorectal and pancreatic cancer for whom palliative chemotherapy was a treatment option, and single interviews with their attending physicians and nurses.

On the basis of our results, we will suggest ways for improving the practise for metastatic cancer patients.

\section{Methods}

We conducted a longitudinal case study of 13 patients with advanced pancreatic or colorectal cancer from an outpatient medical oncology department at a university teaching hospital in the Netherlands. Susanne de Kort (SdK) collected all the data. We observed 60 outpatient physician-patient contacts in the practises of six medical oncologists. During the research period, we also observed weekly team meetings in which physicians and nurses discussed patients. The data from these observations were used in the 30 single semi-structured interviews with the attending health care 
professionals. The main data source was 35 subsequent semi-structured interviews with patients. If there were no treatment changes, the interviews were held every 2 months for pancreatic cancer patients and every 3 months for colorectal cancer patients. Between interviews, SdK stayed in contact by phone at least once a month. A follow-up interview was planned if any changes in the disease course occurred.

A qualitative study design was used because we expected the process leading to a certain treatment course (with or without chemotherapy) to be complex, and we wanted to look for concepts without excluding variables in advance [1].

All patients gave informed consent prior to the start of the study, and during the study the patients were repeatedly told it was possible to stop participating. Patients were asked to give a separate consent to allow us to approach their general practitioner for an interview. The Medical Ethics Committee of the Academic Medical Center in Amsterdam granted a waiver for the study.

\section{Sampling}

Attending physicians included patients with incurable advanced pancreatic or metastatic colorectal cancer (because of a different prognosis related to the chemosensitivity of the tumours, which could influence the decision-making process) who visited their outpatient medical oncology department. Patients with advanced pancreatic cancer have a relatively poor prognosis with a mean survival time of 6 months; chemotherapy has virtually no effect on this [10].

Metastatic colorectal cancer is relatively chemo-sensitive: the prognosis has improved from a mean survival time of 8 months (without treatment) to about 20 months (with a number of types of treatment) [14]. During weekly team meetings, SdK coordinated the selection (purposive sampling) so that patients with different ways of 'deciding' about the cancer treatment ("long-lasting doubters", "refusers", and patients who were willing to have chemotherapy), and equal distribution of cancer types, were included [16].

Between January 2004 and March 2004, 20 patients were selected in the weekly team meetings. Three patients turned out to be ineligible due to a psychiatric problem, an unconfirmed diagnosis, and refusal to cooperate. Four patients refused because of the emotional character of the research subject. In the end, 13 patients were included, and had the following characteristics:

- 7 had pancreatic cancer and 6 had colorectal cancer

- 3 were women and 10 were men

- they had an average age of 64 years (ranging from 41 to 82 years)

- 12 were Caucasian and 1 was Asian

- 4 had an university education and 9 did not

For the semi-structured interviews with health care professionals, we approached different health care professionals who were or had been involved in the treatment of our patient sample. With the exception of one general practitioner, all of them agreed to an interview. Thus, 30 professionals were interviewed between June 2004 and October 2004: eight medical oncologists (two of whom were in training), eight 
general practitioners, three oncology surgeons, three radiotherapists, and eight specialised nurses.

The interviews with the nurses were performed by SdK and Dick Willems (DW) in two focus groups [11]. These groups were organised according to the teams in which they worked.

\section{Interviews}

A total of 35 semi-structured interviews were held: one patient was interviewed once and two patients were interviewed twice (because they died before the second and third interviews, respectively), and the other ten patients were interviewed three times. In the first interview, patients were asked about recent events related to their cancer. Later on, questions focused more on the patients' perceptions of and reasons for certain treatments. The second and third interviews focused on new developments in the disease course, tried to reach a more comprehensive understanding of the themes discussed in the previous interview(s), and discussed patients' treatment decisions. The patient interviews took place in their homes and took about 1-2 $\mathrm{h}$.

The interviews with professionals were held in their offices and took 1-2.5 h. When the health care professionals were interviewed, they were still treating most of the patients; five patients had already died. The interview topics were based on the disease course of the patients who were included and being observed, and addressed the view of the professional. Furthermore, the professionals were invited to talk about other (possibly contrasting) experiences with patients.

\section{Data Analysis}

The unit of analysis was the disease course of 13 patients, with information provided by observations, subsequent interviews with patients, and single interviews with attending physicians and nurses. Interviews were typed out verbatim and coded with MAXqda ${ }^{\circledR}$ software. Recurrent themes were inductively identified through constant comparison, and could be tested by the short cyclic process of analysis and data collection [19]. To increase validity and reliability, the interviewer discussed transcripts and coding with various people (a qualitative researchers forum, a trainee medical student, Jeannette Pols and DW) [12].

In addition to technical matters related to the interviews and data interpretation, the interviewer kept a diary to reflect on her own role and opinions. For example, her initially negative attitude towards continuing chemotherapy until the final weeks of life was challenged when she encountered ideas about living a "good life" and dying while continuing chemotherapy.

\section{Results}

We identified three characteristics of the treatment course in the routine care of patients with metastatic colorectal and pancreatic cancer. These characteristics 
contribute to the idea that the vocabulary of decision-making is not always sufficient and adequate to describe the practise of palliative oncology.

\section{Numerous Options}

We found that the treatment course of metastatic cancer (with or without chemotherapy) was characterised by many options. There were different possibilities with regard to the dose, frequency, and number of chemotherapy courses. Apart from many options while having chemotherapy, there were also many treatment options while having supportive care alone, like trying out a complementary treatment. Patient and physician continuously tailored the treatment in many different ways, based on the patient's condition, his or her experience with treatments, the patient's wishes, and the expected effectiveness of the actual treatment. In the following excerpt from an interview, the frequency of the chemotherapy courses is changed. This is just one of many ways in which treatment can be adapted.

Interviewer: You told me you're taking a bit more time between chemotherapy courses?

Patient 8: Yes, this suits me very well, because we went on vacation for 2 weeks and I did not need to take those pills and everything tasted good again and I could eat everything I liked. Actually, I hadn't felt that well before-I felt weak and very tired.

Interviewer: So do you hope to have a longer interval between courses?

Patient: Yes, for example, one time I can go every 3 weeks and every 4 weeks another time. I'll see.

In this case, the planning of a vacation caused the 3-weekly chemotherapy regimen to be adapted. The patient said she felt much better with this new regimen. Although a standard regimen existed, together with her physician she came up with an alternative, alternating regimen of once every three and then once every 4 weeks. The dose and number of courses as well as the frequency of courses can be adapted in numerous ways. This shows that the practise of palliative chemotherapy is much more complex than the choice with a dilemmic character, namely either palliative chemotherapy or no chemotherapy, that patients might experience. It is more about how much, how often, and how long.

\section{Options Change}

Another remarkable feature of the treatment course was that the nature of the options changed. In contrast to the idea that options are stable-we encountered options that did change identity. In the following interview excerpt, the option of taking a break from chemotherapy gradually changed into stopping completely. The situation Oncologist 2 is talking about is clearly an example of an option that changed identity. Somehow, "tomorrow never came". 
Interviewer asked Oncologist 2 about Patient 2: He hesitated for some time and finally did not have a third cycle of chemotherapy. What made him, or both of you, decide against it?

Oncologist 2: Well, a few things. First of all, he did not have many symptoms from his cancer. And second, he hardly benefited from his earlier cycles of chemotherapy. In fact, they did not do a thing. I also told him I did not expect much. I don't know whether that was decisive for him. From the beginning, he very much agreed with the idea of not starting immediately. And at that time he felt he would start treatment later on. But at a certain moment, this idea had changed. Maybe because he had thought about it himself, or maybe he had discussed it with someone else, I don't know. Although nothing had changed in his health status, somehow he was sure that he wouldn't take the third cycle.

In the excerpt, the interviewer is referring to deciding whether or not to restart the treatment - the pros and cons of this decision would have to be weighed up against each other. But what we observed was different. Nothing was really decisive. A break in the treatment might turn into stopping treatment completely, even if no one made this explicit. Rather than deciding to stop, it just "happened" that the option of "taking a break" turned into "not having any more chemotherapy".

Another example of a change in the nature of an option was observed in the story of Oncologist 8. He described a patient who tried out one treatment and then switched to another one. Instead of weighing up clear treatment options, this switching to a less toxic treatment turned out to be a way of starting to accept the end of life.

Oncologist 8 about a patient (not included in our study) with a tumour that was moderately sensitive to chemotherapy: A patient with a metastatic gastric tumour started with a CEC course [Capecitabine Epirubicine Cysplatin]. I already had my doubts, but he wanted it very much so we decided to evaluate his clinical improvement after one chemotherapy course, after which we would be able to decide again. Then his health deteriorated, but he was not yet ready for the idea of stopping the treatment. We then switched to an oral chemotherapy. He finished one course and then deteriorated again rapidly. I met him once more and at that time we decided we couldn't go on like that.

Interviewer: So that's part of your treatment too: the feeling you have to do something?

Oncologist 8: Yes, I thought such a difficult course of treatment was too much, so we found a compromise with the oral chemotherapy. Although I think I would have preferred to stop, we were talking about something we both had to agree on. This was a reasonable solution.

This patient had a gastric tumour with a poor prognosis and he did not respond well to the first type of chemotherapy. However, there were still numerous treatment options (it was not a question of just "yes or no"). In this specific situation, however, the option of trying out a less toxic treatment turned out to be a way of starting to accept the end of life. According to the oncologist, the patient "was not yet ready for the idea of stopping the treatment". The oral chemotherapy, as an 
"option in-between stopping and continuing treatment", made it possible for the patient to finally stop any treatment aimed at improvement. Treatment options are not always stable and they may change identity.

\section{No Closure}

According to the dictionary definition, a decision is a resolution and hence a closure of a process of consideration (option X means no option Y, Z, etc.). However, in our study the treatment course often involved a continuous process in which the numerous treatment options remain open and can change identities, and in which there is frequently no closure at all. Take the example of "postponing the start of chemotherapy". This may lead to many different activities, such as going on vacation or trying out some alternative treatment. During these activities, the question of whether or not to start chemotherapy is not resolved but remains open. The following excerpt illustrates how numerous options remain.

Patient 10: If hospital X can only offer Gemcitabine [type of chemotherapy], I don't need to decide yet. Nor will I decide if they offer experimental chemonothing has been proven about this yet and the toxic qualities are still being investigated. I don't want to be used as a guinea pig. So I decided to take a month off and now I'm looking around to see what else is available. (...) In fact, I decided I'll have the tumour measured at the beginning of April. If the tumour has gotten substantially smaller, I'll say, don't make changes to a winning team [refers to the alternative medicine he is using at the moment]. But I don't expect that, so it will probably be best to take the next step at that time and to add something. Let's see where it ends up.

During the second interview 2 months later, it emerged that this patient had been actively involved in his treatment course: he continued to use his homoeopathic medicines and had also started a hyperthermia treatment abroad, had visited several allopathic physicians for additional information, and was still not having any chemotherapy. These activities were not preceded by a "closing decision" at one moment but continuously emerged and changed. The different treatment options, including chemotherapy, remained open.

Rather than just starting a treatment, it could be tried out first. This option explicitly implies that the treatment can be stopped. So, taking a treatment does not mean closing off the possibility of having no chemotherapy. Patient 8 told how she tried out a treatment regimen. Although the interviewer and patient both spoke of "decision-making", the excerpt illustrates that the character of the treatment course does not close off all other options, because the possibility of stopping the treatment is included in her considerations.

Interviewer: Do you have the feeling you decided for yourself, that you weighed up the specific advantages and disadvantages connected with continuing another kind of treatment? 
Patient 8: If I were to have the next treatment, I would gain a few months. So I said, Let's go for it. If it makes me very sick, I can always stop. I can at least try it.

Of course, a critical reader could also argue that this quote illustrates that a decision was made, with 'deciding' to start a second cycle of chemotherapy. However, the excerpt also shows that by speaking in terms of "trying out" a treatment, almost all options-like continuing, stopping, and changing the chemotherapy, or the option of switching to another chemotherapy-remain open. In the treatment course of metastatic cancer, options continue to remain open and are not closed as is the implicit idea of 'deciding' in metastatic cancer.

\section{Discussion and Conclusion}

\section{Discussion}

This study has shown that the treatment course of metastatic cancer is more erratic than is easy to describe in straightforward terms used in decision making models. The main findings are:

1. There were many treatment options (including chemotherapy or not).

2. These options were not stable entities to be decided upon, but changed identity over the treatment course.

3. Contrary to the closure (option $\mathrm{X}$ means no option $\mathrm{Y}, \mathrm{Z}$, etc.) a decision implies, the treatment course was a continuous process in which options remained open.

We looked for a metaphor to further clarify the erratic characteristics of the treatment course we encountered in our analysis. Skiing down a mountain has a clear ending at the bottom (any treatment goal) and a skiing instructor or guide (the physician) can accompany you. In off-piste skiing, you have to find your way in the virgin snow. This involves numerous erratic routes that may turn into icy slopes, or a seemingly rough surface may turn out to be a beautiful descent. These routes can be adapted continuously. With touring skis you even might climb back up, so that options remain open. In contrast, decision-based models often look more like deciding for one out of a number of prepared ski slopes.

Mol, in her recent work on care for diabetes patients, uses another metaphor that might be useful [15]. She describes the care provided by health care professionals for patients as a process of trying out, evaluating, and adjusting - in contrast to weighing up and managing by taking decisions. She introduces the concept of "calibration" to describe what professionals and patients do in diabetes care. We believe our findings are quite comparable. However, "off-piste skiing," or "calibration" intuitively seems to fit better with a long-standing physician-patient relationship in a chronic care setting (as in Mol's diabetes care), rather than with those in our study population, who are facing death. We found no evident differences in the erratic treatment course of either pancreatic or colorectal cancer. 
Even in the critical situation of those with advanced pancreatic cancer, the treatment course is characterised by many and changeable options that do not result in closure.

Studies about decision-making in cancer therapy show (1) that not all patients want to participate in the decision-making, $[6,13,18]$ and (2) that they highly value being able to trust their physician [9, 20]. These study results can be better understood in the light of our findings. Patients possibly say they do not want to participate or have the responsibility in decision-making because one-off decisionmaking is rarely the central activity. Added to the complexity of the many changeable options that do not result in closure, trust is possibly the only thing left for patients.

What are the consequences of our data for patient-physician communication models? Of course we could say the practise should be corrected by preventing erratic processes and implementing clear decisions. However, we believe our findings reflect an essential part of the care for the metastatic cancer patient, and therefore current communications models should be adapted. We will discuss the consequences with regard to shared decision making (SDM) which, in our view, is the preferable existing model because of its critical stance to both paternalism and extreme reliance on patient autonomy. SDM is usually advocated as a model for how treatment decisions can-and according to some people, should-be made [2, 3 ]. This type of decision-making is especially associated with treatments for which the benefits are not evident and need to be weighed up against (sometimes considerable) burdens, as is the case in palliative cancer chemotherapy $[4,8,17]$. However, SDM does not reveal anything about the content of decision making, about what kind of decision can be made. In the care for cancer patients SDM is often used as a model for deciding to start or forgo chemotherapy.

\section{Conclusion}

In this study we have shown that some issues in the treatment course of advanced cancer stay underexposed in the current elaborations of the SDM model. Our analysis shows that clear-cut decisions are difficult to pinpoint in the communication between physician and patients about palliative chemotherapy, and that the process leading to a treatment trajectory is often more erratic than decision models allow for. For instance, communication researchers and ethicists should realise that patients and physicians not only deliberate about values attached to fixed treatment options but also create new options about which they deliberate.

Our conclusion could be contested by saying that we used a concept of 'decision' (one-off and conclusive) that is too narrow, and that every disease trajectory may be rewritten as a series of small and at least partly revocable decisions. Even off-piste skiing could be argued to consist of such smaller decisions. However, we believe that this objection would miss the point. Rewriting a disease course, or an off-piste ski run, in terms of decisions is only possible in retrospect, not in the act. Therefore, if it were easily pronounceable, it would be better to speak of shared trajectory making instead of decision making, STM instead of SDM. Just like off-piste tracks are made by sliding, not deciding, disease trajectories are made by events, actions, 
improvisations and negotiations. Incorporating these trajectories is the challenge of SDM.

Our analysis implies a number of ways for improvement of the practise of metastatic cancer treatment. First, the physician has to provide continuous information related to the specific circumstances in time and to the patient's changing situation. When the treatment is a trajectory, because the communication is an ongoing process; the physician needs to be an even better communicator than in the recent SDM model. At the same time, the erratic character of a trajectory removes the demand that all relevant information should be given at one time (the moment of decision-making). To some extent, this would give the physician many opportunities to communicate.

Second, flexibility in dealing with protocols is warranted because the course of a trajectory is not given. For example, treatment protocols should not always be strictly followed. One radiotherapist interviewed put it this way: "Where a cure is concerned, a guideline sets obligations-but the more 'palliative' it becomes, the more reasons-which are also medically imperative-you have not to follow the protocol and to focus on the individual". This flexibility conflicts with the strict research protocols of clinical trials, which causes tension for those patients with metastatic disease who participate in such trials.

Third, vigilance (especially on the part of the physician) about the overall direction of a particular patient trajectory would seem to be of great value because treatment goals are not defined beforehand [5]. The patient and the physician may find themselves in unexpected places. For example, in the situation of Patient 10 (who postponed the decision on whether or not to start chemotherapy), the attending physician also needed to point out the consequences of postponement. If the patient deteriorates rapidly, it could sometimes be too late for this form of treatment.

Ackowledgements We want to thank all of the anonymous respondents for sharing their experiences with us. We would also like to thank Hanneke de Haes, Klaas van Til, Guy Widdershoven, and Myra van Zwieten for their comments on an earlier draft of this manuscript and Nienke van Bergen, who did the coanalysis. Funding: This research project was funded by the Dutch Cancer Society.

Open Access This article is distributed under the terms of the Creative Commons Attribution Noncommercial License which permits any noncommercial use, distribution, and reproduction in any medium, provided the original author(s) and source are credited.

\section{References}

1. Britten, N. (1996). Qualitative interviews in medical research. In N. Mays \& C. Pope (Eds.), Qualitative research in health care. London: BMJ Publishing Group.

2. Charles, C., Gafni, A., \& Whelan, T. (1997). Shared decision-making in the medical encounter: What does it mean? (or it takes at least two to tango). Social Science and Medicine, 44(5), 681-692.

3. de Haes, J. C. J. M. (2006). Dilemmas in patient centeredness and shared decision making: A case for vulnerability. Patient Education and Counseling, 62(3), 291-298.

4. de Haes, J. C. J. M., \& Koedoot, N. (2003). Patient centered decision making in palliative cancer treatment: A world of paradoxes. Patient Education and Counseling, 50(1), 43-49.

5. de Kort, S. J., Willemse, P. H., Habraken, J. M., de Haes, H. C., Willems, D. L., \& Richel, D. J. (2006). Quality of life versus prolongation of life in patients treated with chemotherapy in advanced 
colorectal cancer: A review of randomized controlled clinical trials. European Journal of Cancer, 42(7), 835-845.

6. Degner, L. F., \& Sloan, J. A. (1992). Decision making during serious illness: What role do patients really want to play? Journal of Clinical Epidemiology, 45(9), 941-950.

7. Emanuel, E. J., \& Emanuel, L. L. (1992). Four models of the physician-patient relationship. JAMA: The Journal of the American Medical Association, 267(16), 2221-2226.

8. Gattellari, M., Butow, P. N., \& Tattersall, M. H. (2001). Sharing decisions in cancer care. Social Science and Medicine, 52(12), 1865-1878.

9. Joffe, S., Manocchia, M., Weeks, J. C., \& Cleary, P. D. (2003). What do patients value in their hospital care? An empirical perspective on autonomy centred bioethics. Journal of Medical Ethics, 29(2), 103-108.

10. Kindler, H. L. (2005). Front-line therapy of advanced pancreatic cancer. Seminars in Oncology, $32(6$ Suppl. 9), S33-S36.

11. Kitzinger, J. (1996). Introducing focus groups. In N. Mays \& C. Pope (Eds.), Qualitative research in health care. London: BMJ Publishing group.

12. Lewis, J., \& Ritchie, J. (2003). Generalizing from qualitative research. In J. Lewis \& J. Ritchie (Eds.), Qualitative research practice. London: SAGE Publications.

13. McKinstry, B. (2000). Do patients wish to be involved in decision making in the consultation? A cross sectional survey with video vignettes. BMJ: British Medical Journal, 321(7265), 867-871.

14. Meyerhardt, J. A., \& Mayer, R. J. (2005). Systemic therapy for colorectal cancer. New England Journal of Medicine, 352(5), 476-487.

15. Mol, A. (2008). The logic of care, health and the problem of patient choice. London: Routledge.

16. Ritchie, J., Lewis, J., \& Elam, G. (2003). Designing and selecting samples. In J. Ritchie \& J. Lewis (Eds.), Qualitative research practice: A guide for social science students and researchers (pp. 77108). London: SAGE Publications.

17. Sainio, C., Lauri, S., \& Eriksson, E. (2001). Cancer patients' views and experiences of participation in care and decision making. Nursing Ethics, 8(2), 97-113.

18. Siminoff, L. A., \& Fetting, J. H. (1991). Factors affecting treatment decisions for a life-threatening illness: The case of medical treatment of breast cancer. Social Science and Medicine, 32(7), 813-818.

19. Thorne, S. (2000). Data analysis in qualitative research. Evidence Based Nursing, 3, 68-70.

20. Wright, E. B., Holcombe, C., \& Salmon, P. (2004). Doctors' communication of trust, care, and respect in breast cancer: Qualitative study. BMJ: British Medical Journal, 328(7444), 864. 